\title{
Kernos
}

Revue internationale et pluridisciplinaire de religion grecque antique

$11 \mid 1998$

Varia

\section{Petra PAKKANEN, Interpreting Early Hellenistic Religion. A Study Based on the Mystery Cult of Demeter and the Cult of Isis}

\author{
Michel Malaise
}

\section{OpenEdition \\ Journals}

Édition électronique

URL : http://journals.openedition.org/kernos/1244

DOI : 10.4000/kernos. 1244

ISSN : 2034-7871

Éditeur

Centre international d'étude de la religion grecque antique

Édition imprimée

Date de publication : 1 janvier 1998

ISSN : 0776-3824

Référence électronique

Michel Malaise, «Petra PAKKAnEN, Interpreting Early Hellenistic Religion. A Study Based on the Mystery Cult of Demeter and the Cult of Isis », Kernos [En ligne], 11 | 1998, mis en ligne le 16 juin 2011, consulté le 22 septembre 2020. URL : http://journals.openedition.org/kernos/1244 ; DOI : https://doi.org/ 10.4000/kernos.1244 
Caminos de la muerte), même si cette vision pourrait pécher, du point de vue de Sourvinou-Inwood, par un athénocentrisme réducteur.

En conclusion, Reading Greek Death propose des lectures dont le caractère quelquefois polémique est le reflet d'une richesse de réflexion et d'un souci de dépasser les interprétations antérieures. Une méthodologie qui n'est peut-être pas aussi «neutral » que l'auteur le voudrait, mais qui fonde un ouvrage vraiment intéressant.

Francesco Diez de Velasco

(Universidad de La Laguna - Canarias)

Petra PaKkanen, Interpreting Early Hellenistic Religion. A Study Based on the Mystery Cult of Demeter and the Cult of Isis, Helsinki, 1996. 1 vol. 17,5 $\times 25 \mathrm{~cm}, \mathrm{II}+175 \mathrm{p}$. (Papers and Monographs of the Finnish Institute at Athens, 3). ISBN : 951-95295-4-3.

Le but du présent ouvrage, comme l'expose le chapitre d'introduction, est de réévaluer la mentalité religieuse de l'époque hellénistique en Grèce, à travers le cas d'Athènes, grâce à l'étude de deux cultes, celui de Déméter et celui d'Isis. Le premier, un culte à mystères, établi depuis longtemps à Athènes, constitue un bon représentant des cultes typiques de la polis, offrant à la fois de nombreux éléments individuels et cosmopolites, thèmes qui sont au centre de cette enquête. La religion isiaque, introduite dès avant la fin du iv siècle, livre, elle, un excellent exemple des cultes étrangers pratiqués d'abord au sein d'associations religieuses; elle ne se transformera cependant en religion à mystères qu'a la fin du $i^{\text {er }}$ siècle après J.-C. L'étude est conduite avant tout au départ des sources épigraphiques athéniennes, mais les assez rares témoignages isiaques de la cité athénienne sont complétés par les documents isiaques de Délos.

Dans un second chapitre, l'auteur aborde les problèmes de méthodologie, expliquant qu'elle veut enraciner son étude dans le contexte historique, pour s'ouvrir ensuite à une perspective phénoménologique. Le but recherché est d'expliquer la présence de structures et d'éléments universels dans une religion spécifique, afin de déboucher sur une herméneutique, cherchant par un effort d'interprétation à comprendre le comportement religieux qui amène un individu dans un contexte donné à apporter tel ou tel type de réponse à des pressions sociales, historiques, politiques et économiques.

Le lecteur est ensuite convié dans un troisième chapitre à prendre contact avec une description des cultes choisis. L'auteur souligne d'abord quelques caractéristiques du culte de Déméter : sa continuité dans le temps depuis le $\mathrm{vII}^{\mathrm{e}}$ siècle, son succès dû à son message individuel et émotionnel, mais aussi sa dimension de culte officiel de la Cité et son ouverture à une large participation à partir du III ${ }^{\mathrm{e}}$ siècle. Ensuite, il est question des problèmes d'administration du culte et de son financement tant privé que public. Dans un second temps est dressé un panorama des traits typiques présentés par la religion d'Isis. Débarqué avec des étrangers au Pirée dès avant 333/332, le culte d'Isis est d'abord pratiqué dans le cadre d'associations religieuses, - un phénomène typique de l'époque hellénistique, - au sein desquelles les fidèles se regroupent sur base d'un culte commun et en dehors des structures civiques et familiales, répondant ainsi aux besoins d'une société éclatée. Après une phase de tolérance, puis d'intervention des citoyens dans le culte dès la seconde moitié du III ${ }^{\text {e }}$ siècle, Isis se verra reconnaître un statut officiel vers 200 av. J.-C., sous 'a pression, selon l'auteur, de facteurs politiques (attitude favorable aux Ptolémées) et économiques (nombre grandissant des métèques). Le culte continuera à croître jusqu'au milieu du I $^{\text {er }}$ 
siècle, s'ouvrant aux classes sociales inférieures. Les besoins du culte sont surtout financés par les membres des associations, sans toutefois exclure toute aide officielle.

Le chapitre IV se propose d'étudier le caractère mystérique des cultes de Déméter et d'Isis. Une religion peut être dite à mystères lorsqu'elle procure, par le biais d'une initiation, soigneusement tenue secrète, une union avec la divinité. Durant l'époque hellénistique, le culte éleusinien est une religion à mystères qui réserve une place importante à l'individu, à la pureté et aux préoccupations eschatologiques, traits qui la différencient des autres cultes officiels. Le thiase des dieux égyptiens dans l'Athènes hellénistique réserve aussi une place à l'individu, au cosmopolitisme et à la vie en communauté, cependant il ignore encore la dimension initiatique, introduite avec l'époque romaine. L'initiation isiaque partagera des traits avec le rituel éleusinien, mais elle s'en différenciera par l'existence d'bieroi logoi et l'obligation d'une vie tout entière consacrée à la divinité.

En possession des éléments recueillis à travers l'étude de ces deux cultes, l'A. tente enfin de réévaluer les concepts caractérisant la religion hellénistique, et d'abord celui du syncrétisme. Dans une première phase, les figures de Déméter et d'Isis sont simplement juxtaposées. Ensuite, les analogies présentées par les deux déesses mènent à une phase de parallélisme, qui conduit enfin à une véritable identification; cette phase est exprimée clairement pour la première fois dans l'arétalogie de Maronée et est contemporaine de l'introduction des mystères d'Isis. Ce syncrétisme favorisera la naissance d'une tendance hénothéiste puis monothéiste, certaines divinités recelant en elles les diverses manifestations du pouvoir divin. L'individualisme n'est pas une nouveauté totale de l'époque hellénistique, mais l'éclatement de la polis, le caractère plus cosmopolite de la Cité, - imposé par la nécessité d'une ouverture politique et économique, - et la réflexion philosophique favorisent le regroupement d'individus au sein d'associations cultuelles, en dehors des anciennes restrictions ethniques et de la religion de la Cité. La tendance monothéiste est aussi favorable à l'épanouissement de la religiosité personnelle parce que la divinité rencontrée jouit d'un pouvoir autocratique qui amène le fidèle à se tourner vers de tels dieux capables de procurer le salut.

L'ouvrage ici résumé nous semble présenter un certain décalage entre son propos et ses résultats. Si le but annoncé par l'A. est de se faire une idée de la mentalité religieuse à l'épreuve des faits replacés dans un contexte historique, force est de constater que son propos dévie souvent en considérations générales et abstraites qui n'apportent rien de neuf à la compréhension profonde de la religion hellénistique. Sans doute le choix restreint de deux cultes offrait-il trop peu de faits concrets pour éviter cet écueil. En outre, il apparaît que la culture isiaque et égyptologique de l'A. n'est pas toujours à la hauteur de son propos. Cela ressort notamment des lacunes bibliographiques. Ainsi, bien que l'initiation isiaque soit un des points essentiels abordés, des articles fondamentaux sont omis, comme ceux de J. Assmann, J. Bergman, E. Hornung, F. Junge, L. Kakosy et E. WeNTE, pour ne citer que ceux-là. À propos des arétalogies, il est faux de considérer que l'Isis thesmophoros et civilisatrice s'explique par des antécédents égyptiens; par ailleurs, sur cette question, il était important de tirer parti de l'étude de L. ZaBkar, Hymns to Isis in Her Temple at Philae, 1989. Tout aussi contestable est l'assertion suivant laquelle l'Isis débarquée à Athènes serait clairement distincte de Déméter; c'est là faire peu de cas de tout le travail d'hellénisation qui s'est déroulé sur le sol de l'Égypte grecque. Quant au portrait de l'Isis égyptienne, il n'est guère heureux: Isis n'est pas d'abord une déesse solaire, et l'image qui en est donnée est déjà fortement hellénisée. Plus grave est la confusion qui amène l'A. (p. 78) à considérer la ciste comme « mystery or 
canopic jar », confondant ainsi la corbeille sacrée, pourtant attestée aussi en dehors des cultes isiaques, avec l'hydrie et les Osiris-Canope; sur ce mobilier rituel, on nous permettra de renvoyer à notre étude Ciste et bydrie, symboles de la puissance et de la présence d'Osiris, in J. RiEs (éd.), Le symbolisme dans le culte des grandes religions. Actes du Colloque de Louvain-la-Neuve 4-5 octobre 1983, Louvain-la Neuve, 1985 , p. 125-155. Sur le problème de l'introduction du culte d'Isis à Athènes, on aurait voulu voir citer la référence à l'article de R. Simms, Isis in classical Athens, in CJ, 84 (1988-89), p. 216-221.

En résumé, si les ambitions affichées par l'A. dans ses propos théoriques sont alléchantes, on ne peut dire que cet ouvrage apporte un éclairage vraiment neuf ni sur les cultes de Déméter et d'Isis, ni sur la religion hellénistique.

Michel Malaise (Université de Liège)

Rebeca Rubio (éd.), Isis. Nuevas perspectivas. Homenanje al Prof. Alvarez de Miranda, Madrid, Ediciones Clásicas, 1996. 1 vol. $15,5 \times 21,5 \mathrm{~cm}, \mathrm{X}+155$ p. (Collection "Arys" = Antigüedad: Religiones y Sociedades, 4).

Ce volume nous offre le texte de 11 études isiaques présentées lors d'un colloque tenu voici déjà plusieurs années, les 30 et 31 mai 1988. La plupart des communications traitent de questions relatives au problème de la diffusion isiaque à travers le monde antique. Si, en Afrique du Nord, la pénétration des cultes égyptiens s'est parfois produite avant l'époque hellénistique, grâce à des contacts directs avec l'Égypte (comme dans le cas de Carthage), R. CrF, El culto a "Isis" en Numidia: los testiminios del campamento militar de "Lambaesis" (p. 47 63) souligne que les conditions de diffusion de ces mêmes cultes en Numidie sont assez différentes, dans la mesure où Isis et Sérapis s'y implantent assez tard, et avant tout dans les secteurs les plus romanisés de la société provinciale. En revanche, J. Alvar, Isis prerromana, Isis romana (p. 95-107) montre que l'introduction des cultes isiaques dans la péninsule ibérique à l'époque romaine est un phénomène nouveau qui ne constitue en rien un prolongement de la présence de documents égyptiens ou égyptisants débarqués bien auparavant dans la péninsule grâce aux commerçants phéniciens. Le cas de la Gaule Narbonnaise est analysé par R. Siera, Isis en la Galia Narbonense; nuevas perspectivas (p. 123 134), lequel montre que les cultes isiaques s'y sont répandus, en suivant le couloir rhodanien, à travers toutes les couches sociales, mais avant tout dans les milieux urbains; l'absence d'indigènes parmi les sectateurs fournit un indice que cette diffusion n'est pas davantage à expliquer par des contacts d'époque préromaine. Pour la Bretagne romaine, R. RuBIo, El culto de Isis y Serapis en Britania (p. 35-46) repasse en revue les témoignages isiaques découverts sur l'île afin de mesurer l'impact géographique et social de ces cultes, constatant à nouveau leur enracinement dans les cercles romanisés, et leur absence tant dans les classes inférieures de la société que dans le milieu indigène; Isis étant surtout présente dans le Sud du pays, tandis que Sérapis est surtout lié aux établissements militaires du Nord de l'île. L'implantation d'Isis en certains lieux peut être mise en relation avec ses vertus de déesse guérisseuse; c'est pourquoi F. DIEz DE VELASCO, Invocaciones a Isis en cividades de aguas (Aquae) del Occidente romano (p. 143-153) souligne que la déesse égyptienne est parfois invoquée conjointement avec Hygeia ou les Nymphes, et relève la présence d'Isis dans un certain nombre de cités thermales où la déesse égyptienne a pu dispenser ses guérisons au travers des eaux curatives. Selon D. PLÁCIDo, El culto de Isis en Atenas durante el Imperio Romano (p. 1-11), Athènes fut un des lieux où le culte 